\title{
Solution and intuitionistic fuzzy stability of 3- dimensional cubic functional equation: using two different methods
}

\author{
Jyotsana Jakhara ${ }^{a}$, Renu Chugh ${ }^{a}$, Jagjeet Jakhar ${ }^{\mathrm{b}, *}$ \\ a Department of Mathematics, M.D. University, Rohtak-124001, Haryana, India. \\ ${ }^{b}$ Department of Mathematics, Central University of Haryana, Mahendergarh-123031, Haryana, India.
}

\begin{abstract}
In this article, we adopt fixed point method and direct method to find the solution and Intuitionistic fuzzy stability of 3dimensional cubic functional equation

$$
\begin{aligned}
g\left(2 u_{1}+u_{2}+u_{3}\right)= & 3 g\left(u_{1}+u_{2}+u_{3}\right)+g\left(-u_{1}+u_{2}+u_{3}\right)+2 g\left(u_{1}+u_{2}\right)+2 g\left(u_{1}+u_{3}\right)-6 g\left(u_{1}-u_{2}\right)-6 g\left(u_{1}-u_{3}\right) \\
& -3 g\left(u_{2}+u_{3}\right)+2 g\left(2 u_{1}-u_{2}\right)+2 g\left(2 u_{1}-u_{3}\right)-18 g\left(u_{1}\right)-6 g\left(u_{2}\right)-6 g\left(u_{3}\right)
\end{aligned}
$$
\end{abstract}

Keywords: Functional equations, intuitionistic fuzzy Banach space, fixed point method, direct method.

2020 MSC: 39B52, 39B82, 46S40.

(C)2022 All rights reserved.

\section{Introduction}

The first stability problem was established by Ulam [29] in 1940. He raised a question "Is there an exact homomorphism close to approximate homomorphism?". An answer of Ulam problem was given by Hyers [15] in Banach spaces. Since then, the stability problems have been studied by several mathematicians.

In 1965, Zadeh [30] initialized the theory of fuzzy sets. Through the classical learning of Zadeh, there has been a large work to find fuzzy illustration of academic notions. Later on, various generalizations and extensions of Hyers' result were established by Rassias [22, 23], Găvruta [13], and Rassias [24] in different versions for the Cauchy (additive) functional equation $f(x+y)=f(x)+f(y)$ in Banach spaces. Since then, the stability problems of various functional equations on miscellaneous normed spaces have been extensively investigated by a number of authors; for instance, see $[1-5,7,8,11]$.

Authors $[4,9,21]$ pointed out that a fixed point alternative method can be successfully used to solve the Ulam problem. The fixed point method in the study of the stability of functional equations firstly appears

\footnotetext{
*Corresponding author

Email addresses: dahiya.jyotsana.j@gmail.com (Jyotsana Jakhar), chugh.r1@gmail.com (Renu Chugh), jagjeet@cuh.ac.in (Jagjeet Jakhar)
}

doi: $10.22436 /$ jmcs.025.02.01

Received: 2021-02-27 Revised: 2021-03-24 Accepted: 2021-04-15 
in [12]. In order to have more knowledge on the various stability problems of functional equations see [18-20, 25-27]. Jun et al. [16] introduced the following functional equation

$$
g(2 u+v)+g(2 u-v)=2 g(u+v)+2 g(u-v)+12 g(u)
$$

and explored its ordinary solution and Hyers-Ulam stability respectively. The functional equation (1.1) is called cubic since $\mathrm{g}(\mathrm{u})=\mathrm{cu}^{3}$ is its solution. Many authors introduced the new types of functional equations which is cubic and one of the type of 3-dimensional cubic functional equation

$$
\begin{aligned}
g\left(2 u_{1}+u_{2}+u_{3}\right)= & 3 g\left(u_{1}+u_{2}+u_{3}\right)+g\left(-u_{1}+u_{2}+u_{3}\right)+2 g\left(u_{1}+u_{2}\right) \\
& +2 g\left(u_{1}+u_{3}\right)-6 g\left(u_{1}-u_{2}\right)-6 g\left(u_{1}-u_{3}\right)-3 g\left(u_{2}+u_{3}\right) \\
& +2 g\left(2 u_{1}-u_{2}\right)+2 g\left(2 u_{1}-u_{3}\right)-18 g\left(u_{1}\right)-6 g\left(u_{2}\right)-6 g\left(u_{3}\right)
\end{aligned}
$$

and introduced by Park et al. [14].

Preliminaries and definitions

Definition 1.1 ([10]). Let $\mathrm{L}^{*}$ be any set and the order relation $\leqslant \mathrm{L}^{*}$ defined by

$$
\begin{gathered}
\mathrm{L}^{*}=\left\{\left(\mathrm{u}_{1}, \mathrm{u}_{2}\right):\left(\mathrm{u}_{1}, \mathrm{u}_{2}\right) \in[0,1]^{2} \text { and } \mathrm{u}_{1}+\mathrm{u}_{2} \leqslant 1\right\}, \\
\left(\mathrm{u}_{1}, \mathrm{u}_{2}\right) \leqslant \mathrm{L}^{*}\left(v_{1}, v_{2}\right) \Leftrightarrow \mathrm{u}_{1} \leqslant v_{1}, \mathrm{u}_{2} \geqslant v_{2}, \quad \forall\left(\mathrm{u}_{1}, \mathrm{u}_{2}\right),\left(v_{1}, v_{2}\right) \in \mathrm{L}^{*} .
\end{gathered}
$$

Then the ordered pair $\left(\mathrm{L}^{*}, \leqslant \mathrm{~L}^{*}\right)$ is a complete lattice.

Definition $1.2([6])$. An intuitionistic fuzzy set $\mathcal{A}_{\sigma, v}$ in a universal set $\mathcal{U}$ is an object

$$
\mathcal{A}_{\sigma, v}=\left\{\left(\sigma_{\mathcal{A}}(\mathrm{u}), \mathfrak{v}_{\mathcal{A}}(\mathrm{u})\right) \mid \mathrm{u} \in \mathcal{U}\right\}
$$

for all $u \in \mathcal{U}, \sigma_{\mathcal{A}}(u) \in[0,1]$ and $v_{\mathcal{A}}(u) \in[0,1]$ are called the membership degree and the non-membership degree, respectively, of $u$ in $\mathcal{A}_{\sigma, v}$ and furthermore, they satisfy $\sigma_{\mathcal{A}}(u)+v_{\mathcal{A}}(u) \leqslant 1$. We denote its units by $0_{\mathrm{L}^{*}}=(0,1)$ and $1_{\mathrm{L}^{*}}=(1,0)$.

Definition $1.3([6])$. A triangular norm ( $t^{\prime}$-norm) on $L^{*}$ is a mapping $\mathcal{T}:\left(L^{*}\right)^{2} \rightarrow L^{*}$ satisfying the following

- (boundary condition): $\mathcal{T}\left(\mathfrak{u}, 1_{\mathrm{L}^{*}}\right)=\mathfrak{u}$;

- (commutativity): $\mathcal{T}(u, v)=\mathcal{T}(v, u)$;

- (associativity): $\mathcal{T}(u, \mathcal{T}(v, w))=\mathcal{T}(\mathcal{T}(u, v), w)$;

- (monotonicity): $u \leqslant_{\mathrm{L}^{*}} \mathrm{u}^{\prime}$ and $v \leqslant_{\mathrm{L}^{*}} v^{\prime} \Rightarrow \mathcal{T}(\mathrm{u}, v) \leqslant \mathrm{L}^{*} \mathcal{T}\left(\mathrm{u}^{\prime}, v^{\prime}\right)$.

If $\left(\mathrm{L}^{*}, \mathrm{~L}^{*}, \mathcal{T}\right)$ is an Abelian topological monoid with unit $1_{\mathrm{L}^{*}}$, then $\mathrm{L}^{*}$ is said to be a continuous $\mathrm{t}^{\prime}$-norm.

Definition 1.4 ([28]). A negation on $\mathrm{L}^{*}$ is any decreasing mapping $\mathcal{N}: \mathrm{L}^{*} \rightarrow \mathrm{L}^{*}$ satisfying $\mathcal{N}\left(0_{\mathrm{L}^{*}}\right)=1_{\mathrm{L}^{*}}$ and $\mathcal{N}\left(1_{\mathrm{L}^{*}}\right)=0_{\mathrm{L}^{*}}$. If $\mathcal{N}(\mathcal{N}(u))=u$ for all $\mathrm{u} \in \mathrm{L}^{*}$, then $\mathcal{N}$ is called an involutive negator.

Definition 1.5 ([28]). Let $\alpha$ and $\beta$ be membership and non-membership degree of an intuitionistic fuzzy set from $\mathrm{U} \times(0, \infty)$ to $[0,1]$ such that $\alpha_{\mathfrak{u}}\left(t^{\prime}\right)+\beta_{\mathfrak{u}}\left(t^{\prime}\right) \leqslant 1$ for all $u \in U$ and $t^{\prime}>0$. The triple $(U, \mathcal{P} \alpha, \beta, \mathcal{T})$ is said to be an intuitionistic fuzzy normed space (IFNS) if $U$ is a vector space, $\mathcal{T}$ is a continuous $t^{\prime}$ representable and $\mathcal{P}_{\alpha, \beta}$ is a mapping $\mathrm{U} \times(0, \infty) \rightarrow \mathrm{L}^{*}$ satisfying the following

IFN1. $\mathcal{P}_{\alpha, \beta}(u, 0)=0_{L^{*}} ;$

IFN2. $\mathcal{P}_{\alpha, \beta}\left(u, t^{\prime}\right)=1_{L^{*}} \Leftrightarrow u=0$;

IFN3. $\mathcal{P}_{\alpha, \beta}\left(\mathrm{cu}, \mathrm{t}^{\prime}\right)=\mathcal{P}_{\alpha, \beta}\left(\mathrm{u}, \frac{\mathrm{t}^{\prime}}{\|\mathrm{c}\|}\right)$ for all $\mathrm{c} \neq 0$;

IFN4. $\mathcal{P}_{\alpha, \beta}\left(u+v, t^{\prime}+s^{\prime}\right) \geqslant_{L^{*}} \mathcal{T}\left(\mathcal{P}_{\alpha, \beta}\left(u, t^{\prime}\right), \mathcal{P}_{\alpha, \beta}\left(v, s^{\prime}\right)\right)$ for all $u, v \in U$ and $t^{\prime}, s^{\prime}>0$. 
In this case, $\mathcal{P}_{\alpha, \beta}$ is called an intuitionistic fuzzy norm. Here, $\mathcal{P}_{\alpha, \beta}\left(u, t^{\prime}\right)=\left(\alpha_{\mathfrak{u}}\left(t^{\prime}\right), \beta_{\mathfrak{u}}\left(t^{\prime}\right)\right)$.

Definition $1.6([28])$. A sequence $\left\{u_{m}\right\}$ in an IFNS $\left(U, \mathcal{P} \mathcal{P}_{\alpha, \beta}, \mathcal{T}\right)$ is called a Cauchy sequence if for any $\varepsilon>0$ and $t^{\prime}>0$, there exists $m_{0} \in \mathcal{N}$ such that

$$
\mathcal{P}_{\alpha, \beta}\left(u_{m}-u_{m^{\prime}}, t^{\prime}\right)>L^{*}\left(\mathcal{N}_{s}(\varepsilon), \varepsilon\right), \forall m, m^{\prime} \geqslant m_{0},
$$

where $\mathcal{N}_{s}$ is the standard negator. The sequence $\left\{u_{m}\right\}$ is said to be convergent to a point $u \in U$ if

$$
\mathcal{P}_{\alpha, \beta}\left(u_{m}-u, t^{\prime}\right) \rightarrow 1_{L^{*}}
$$

as $m \rightarrow \infty$ for every $t^{\prime}>0$. An IFNS $\left(U, \mathcal{P}_{\alpha, \beta}, \mathcal{T}\right)$ is said to be complete if every cauchy sequence in $U$ is convergent to a point $u \in U$.

In this article, we adopt fixed point method and direct method to find the solution and Intuitionistic fuzzy stability of 3-dimensional cubic functional equation (1.2) in intuitionistic fuzzy normed space (IFNS).

\section{General solution}

In this segment, we find the solution of the functional equation (1.2) in intuitionistic fuzzy normed spaces.

Theorem 2.1. Let $\mathrm{U}$ and $\mathrm{V}$ be real vector spaces. The mapping $\mathrm{g}: \mathrm{U} \rightarrow \mathrm{V}$ satisfies the functional equation (1.1) for all $\mathrm{u}, v \in \mathrm{U}$ iff $\mathrm{g}: \mathrm{U} \rightarrow \mathrm{V}$ satisfies the functional equation (1.2) for all $\mathrm{u}_{1}, \mathrm{u}_{2}, \mathrm{u}_{3} \in \mathrm{U}$.

Proof. Let $\mathrm{g}: \mathrm{U} \rightarrow \mathrm{V}$ satisfies the functional equation (1.1). Putting $(u, v)=(0,0)$ in (1.1), we get $g(0)=0$. Substituting $(u, v)=(0, v)$ in $(1.1)$, we get

$$
g(-v)=-g(v)
$$

for all $v \in \mathrm{U}$. Therefore $g$ is an odd function. Replacing $(u, v)$ by $(u, 0),(u, u)$, and $(u, 2 u)$, respectively in (1.1), we get

$$
g(2 u)=2^{3} g(u), g(3 u)=3^{3} g(u), \text { and } g(4 u)=4^{3} g(u)
$$

for all $u \in U$. In general for any positive integer $c$, we have

$$
g(c u)=c^{3} g(u)
$$

for all $u \in U$. Putting $(u, v)=\left(u_{1}, u_{2}+u_{3}\right)$ in (1.1), we get

$$
g\left(2 u_{1}+u_{2}+u_{3}\right)-2 g\left(u_{1}+u_{2}+u_{3}\right)=g\left(-2 u_{1}+u_{2}+u_{3}\right)+2 g\left(u_{1}-u_{2}-u_{3}\right)+12 g\left(u_{1}\right) .
$$

Again putting $(u, v)=\left(u_{2}+u_{3},-2 u_{1}\right)$ in (1.1), we get

$$
4 g\left(-u_{1}+u_{2}+u_{3}\right)+4 g\left(u_{1}+u_{2}+u_{3}\right)-g\left(2 u_{1}+u_{2}+u_{3}\right)-6 g\left(u_{2}+u_{3}\right)=g\left(-2 u_{1}+u_{2}+u_{3}\right)
$$

for all $u_{1}, u_{2}, u_{3} \in U$. Substituting (2.2) in (2.1), we get

$$
g\left(2 u_{1}+u_{2}+u_{3}\right)-3 g\left(u_{1}+u_{2}+u_{3}\right)=g\left(-u_{1}+u_{2}+u_{3}\right)-3 g\left(u_{2}+u_{3}\right)+6 g\left(u_{1}\right) .
$$

Now putting $(u, v)=\left(u_{2}, 2 u_{1}\right)$ in $(1.1)$, we get

$$
4 g\left(u_{1}+u_{2}\right)-4 g\left(u_{1}-u_{2}\right)-6 g\left(u_{2}\right)=g\left(2 u_{1}+u_{2}\right)-g\left(2 u_{1}-u_{2}\right) .
$$


Replacing $(u, v)$ by $\left(u_{3}, 2 u_{1}\right)$ in $(1.1)$, we get

$$
4 g\left(u_{1}+u_{3}\right)-4 g\left(u_{1}-u_{3}\right)-6 g\left(u_{3}\right)=g\left(2 u_{1}+u_{3}\right)-g\left(2 u_{1}-u_{3}\right) .
$$

Adding (2.4) and (2.5), we get

$$
\begin{aligned}
& 4 g\left(u_{1}+u_{2}\right)-4 g\left(u_{1}-u_{2}\right)+4 g\left(u_{1}+u_{3}\right)-4 g\left(u_{1}-u_{3}\right)-6 g\left(u_{2}\right)-6 g\left(u_{3}\right) \\
& -g\left(2 u_{1}+u_{2}\right)+g\left(2 u_{1}-u_{2}\right)-g\left(2 u_{1}+u_{3}\right)+g\left(2 u_{1}-u_{3}\right)=0 .
\end{aligned}
$$

Again adding (2.3) and (2.6), we get

$$
\begin{aligned}
g\left(2 u_{1}+u_{2}+u_{3}\right)= & 3 g\left(u_{1}+u_{2}+u_{3}\right)+g\left(-u_{1}+u_{2}+u_{3}\right) \\
& -3 g\left(u_{2}+u_{3}\right)+6 g\left(u_{1}\right)+4 g\left(u_{1}+u_{2}\right)-4 g\left(u_{1}-u_{2}\right) \\
& +4 g\left(u_{1}+u_{3}\right)-4 g\left(u_{1}-u_{3}\right)-6 g\left(u_{2}\right)-6 g\left(u_{3}\right)-g\left(2 u_{1}+u_{2}\right) \\
& +g\left(2 u_{1}-u_{2}\right)-g\left(2 u_{1}+u_{3}\right)+g\left(2 u_{1}-u_{3}\right) .
\end{aligned}
$$

Replacing $(u, v)$ by $\left(-u_{1}, u_{2}\right)$ in (1.1), we get

$$
-g\left(2 u_{1}+u_{2}\right)=g\left(2 u_{1}-u_{2}\right)-2 g\left(u_{1}-u_{2}\right)-2 g\left(u_{1}+u_{2}\right)-12 g\left(u_{1}\right) .
$$

Replacing $(u, v)$ by $\left(-u_{1}, u_{3}\right)$ in $(1.1)$, we get

$$
-g\left(2 u_{1}+u_{3}\right)=g\left(2 u_{1}-u_{3}\right)-2 g\left(u_{1}-u_{3}\right)-2 g\left(u_{1}+u_{3}\right)-12 g\left(u_{1}\right) .
$$

Adding (2.8) and (2.9), we obtain

$$
\begin{aligned}
-g\left(2 u_{1}+u_{2}\right)-g\left(2 u_{1}+u_{3}\right)= & g\left(2 u_{1}-u_{2}\right)-2 g\left(u_{1}-u_{2}\right)-2 g\left(u_{1}+u_{2}\right)-2 g\left(u_{1}-u_{3}\right) \\
& +g\left(2 u_{1}-u_{3}\right)-2 g\left(u_{1}-u_{3}\right)-g\left(u_{1}+u_{3}\right)-24 g\left(u_{1}\right) .
\end{aligned}
$$

Substituting (2.10) in (2.7), we have

$$
\begin{aligned}
g\left(2 u_{1}+u_{2}+u_{3}\right)= & 3 g\left(u_{1}+u_{2}+u_{3}\right)+g\left(-u_{1}+u_{2}+u_{3}\right)+2 g\left(u_{1}+u_{2}\right)+2 g\left(u_{1}\right. \\
& \left.+u_{3}\right)-6 g\left(u_{1}-u_{2}\right)-6 g\left(u_{1}-u_{3}\right)-3 g\left(u_{2}+u_{3}\right)+2 g\left(2 u_{1}-u_{2}\right) \\
& +2 g\left(2 u_{1}-u_{3}\right)-18 g\left(u_{1}\right)-6 g\left(u_{2}\right)-6 g\left(u_{3}\right)
\end{aligned}
$$

for all $u_{1}, u_{2}, u_{3} \in U$. Conversely, let $g: U \rightarrow V$ satisfy the functional equation (1.2). Substituting $\left(\mathfrak{u}_{1}, \mathfrak{u}_{2}, \mathfrak{u}_{3}\right)=\left(0, \mathfrak{u}_{2}, 0\right)$ in $(1.2)$, we get

$$
g\left(-u_{2}\right)=-g\left(u_{2}\right)
$$

for all $u_{2} \in U$. Therefore $g$ is an odd function. Replacing $\left(u_{1}, u_{2}, u_{3}\right)$ by $(u, v, 0)$ in $(1.2)$, we have

$$
g(2 u+v)-2 g(2 u-v)=5 g(u+v)-7 g(u-v)-6 g(u)-9 g(v) .
$$

Again replacing $\left(\mathfrak{u}_{1}, \mathfrak{u}_{2}, \mathfrak{u}_{3}\right)$ by $(u, 0,-v)$ in $(1.2)$, we have

$$
g(2 u-v)-2 g(2 u+v)=-7 g(u+v)+5 g(u-v)-6 g(u)+9 g(v)
$$

for all $u, v \in U$. Adding (2.11) and (2.12), we get our results. 


\section{Stability results}

In this segment, we present the generalized Ulam-Hyers stability of the functional equation (1.2) in intuitionistic fuzzy normed spaces using direct method. Now, we use the following notation for a given mapping $\mathrm{g}: \mathrm{U} \rightarrow \mathrm{V}$

$$
\begin{aligned}
\operatorname{Dg}\left(u_{1}, u_{2}, u_{3}\right)= & g\left(2 u_{1}+u_{2}+u_{3}\right)-3 g\left(u_{1}+u_{2}+u_{3}\right)-g\left(-u_{1}+u_{2}+u_{3}\right) \\
& -2 g\left(u_{1}+u_{2}\right)-2 g\left(u_{1}+u_{3}\right)+6 g\left(u_{1}-u_{2}\right)+6 g\left(u_{1}-u_{3}\right)+3 g\left(u_{2}+u_{3}\right) \\
& -2 g\left(2 u_{1}-u_{2}\right)-2 g\left(2 u_{1}-u_{3}\right)+18 g\left(u_{1}\right)+6 g\left(u_{2}\right)+6 g\left(u_{3}\right) .
\end{aligned}
$$

Theorem 3.1. Let $\gamma \in(-1,1)$. Let $\mathrm{U}$ be a linear space, $\left(Z, \mathcal{P}^{\prime}{ }_{\alpha, \beta}, \mathcal{T}\right)$ be an IFNS, $\mathrm{h}: \mathrm{U}^{3} \rightarrow Z$ be a mapping with $0<\left(\frac{\tau}{2^{3}}\right)^{\gamma}<1$, for some $\tau>0$,

$$
\mathcal{P}^{\prime}{ }_{\alpha, \beta}\left(\mathrm{h}\left(2^{\gamma} \mathrm{u}, 0,0\right), \varepsilon\right) \geqslant_{\mathrm{L}^{*}} \mathcal{P}^{\prime}{ }_{\alpha, \beta}\left(\tau^{\gamma} \mathrm{h}(\mathrm{u}, 0,0), \varepsilon\right)
$$

for all $\mathrm{u} \in \mathrm{U}$ and $\varepsilon>0$ and

$$
\lim _{m \rightarrow \infty} \mathcal{P}_{\alpha, \beta}^{\prime}\left(h\left(2^{\gamma m} u_{1}, 2^{\gamma m} u_{2}, 2^{\gamma m} u_{3}\right), 2^{\gamma 3 m} \varepsilon\right)=1_{L^{*}}
$$

for all $\mathrm{u}_{1}, \mathrm{u}_{2}, \mathrm{u}_{3} \in \mathrm{U}$ and $\varepsilon>0$. Assume that a mapping $\mathrm{g}: \mathrm{U} \rightarrow \mathrm{V}$ satisfies the inequality

$$
\mathcal{P}_{\alpha, \beta}\left(\mathrm{Dg}\left(\mathrm{u}_{1}, \mathrm{u}_{2}, \mathrm{u}_{3}\right), \varepsilon\right) \geqslant_{\mathrm{L}^{*}} \mathcal{P}^{\prime}{ }_{\alpha, \beta}\left(\mathrm{h}\left(\mathrm{u}_{1}, \mathrm{u}_{2}, \mathrm{u}_{3}\right), \varepsilon\right) .
$$

Then the limit

$$
\mathcal{P}_{\alpha, \beta}\left(\mathrm{Q}(\mathrm{u})-\frac{\mathrm{g}\left(2^{\gamma \mathrm{m}} \mathrm{u}\right)}{2^{\gamma 3 \mathrm{~m}}}\right) \longrightarrow 1_{\mathrm{L}^{*}} \text { as } \mathrm{m} \rightarrow \infty, \varepsilon>0
$$

exists for all $\mathrm{u} \in \mathrm{U}$ and the mapping $\mathrm{Q}: \mathrm{U} \rightarrow \mathrm{V}$ is a unique cubic mapping satisfying (1.2) and

$$
\mathcal{P}_{\alpha, \beta}(g(u)-Q(u), \varepsilon) \geqslant_{L^{*}}\left(h(u, 0,0), 3 \varepsilon\left|2^{3}-\tau\right|\right)
$$

for all $\mathrm{u} \in \mathrm{U}$ and $\varepsilon>0$.

Proof. Firstly, assume $\gamma=1$. Replacing $\left(\mathfrak{u}_{1}, \mathfrak{u}_{2}, \mathfrak{u}_{3}\right)$ by $(\mathfrak{u}, 0,0)$ in (3.3), we get

$$
\mathcal{P}_{\alpha, \beta}(3 g(2 u)-24 g(u), \varepsilon) \geqslant L^{*} \mathcal{P}^{\prime}{ }_{\alpha, \beta}(h(u, 0,0), \varepsilon)
$$

for all $u \in U$ and $\varepsilon>0$. Replacing $u$ by $2^{m} u$ in (1.2) and using (IFN3), we have

$$
\mathcal{P}_{\alpha, \beta}\left(\frac{g\left(2^{m+1} u\right)}{2^{3}}-g\left(2^{m} u\right), \frac{\varepsilon}{24}\right) \geqslant L^{*} \mathcal{P}^{\prime}{ }_{\alpha, \beta}\left(h\left(2^{m} u, 0,0\right), \varepsilon\right) .
$$

Again using (3.1), (IFN3) in (3.5), we find

$$
\mathcal{P}_{\alpha, \beta}\left(\frac{g\left(2^{m+1} \mathfrak{u}\right)}{2^{3}}-g\left(2^{m} u\right), \frac{\varepsilon}{24}\right) \geqslant L^{*} \mathcal{P}^{\prime}{ }_{\alpha, \beta}\left(h(u, 0,0), \frac{\varepsilon}{\tau^{m}}\right) .
$$

It is simple to check from (3.6), that

$$
\mathcal{P}_{\alpha, \beta}\left(\frac{g\left(2^{m+1} \mathfrak{u}\right)}{2^{3(m+1)}}-\frac{g\left(2^{m} \mathfrak{u}\right)}{2^{3 m}}, \frac{\varepsilon}{24.2^{3 m}}\right) \geqslant \mathrm{L}^{*} \mathcal{P}^{\prime}{ }_{\alpha, \beta}\left(h(u, 0,0), \frac{\varepsilon}{\tau^{m}}\right)
$$

holds for all $u \in U$ and $\varepsilon>0$. Replacing $\varepsilon$ by $\tau^{m} \varepsilon$ in (3.7), we obtain

$$
\mathcal{P}_{\alpha, \beta}\left(\frac{g\left(2^{m+1} u\right)}{2^{3(m+1)}}-\frac{g\left(2^{m} u\right)}{2^{3 m}}, \frac{\varepsilon \tau^{m}}{24.2^{3 m}}\right) \geqslant L^{*} \mathcal{P}^{\prime}{ }_{\alpha, \beta}(h(u, 0,0), \varepsilon)
$$


for all $u \in U$ and $\varepsilon>0$. It is simple to see that

$$
\frac{g\left(2^{m} u\right)}{2^{3 m}}-g(u)=\sum_{i=0}^{m-1} \frac{g\left(2^{i+1} u\right)}{2^{3(i+1)}}-\frac{g\left(2^{i} u\right)}{2^{3 i}}
$$

for all $u \in U$. From equations (3.8) and (3.9), we get

$$
\begin{aligned}
\mathcal{P}_{\alpha, \beta}\left(\frac{g\left(2^{m} \mathfrak{u}\right)}{2^{3 m}}-g(u), \Sigma_{i=0}^{m-1} \frac{\tau^{i} \varepsilon}{3\left(2^{3 i} \cdot 2^{3}\right)}\right) & \geqslant_{L^{*}} \mathcal{T}_{i=0}^{m-1}\left\{\mathcal{P}_{\alpha, \beta}^{\prime}\left(\frac{g\left(2^{i+1} u\right)}{2^{3(i+1)}}-\frac{g\left(2^{i} u\right)}{2^{3 i}}, \frac{\tau^{i} \varepsilon}{3\left(2^{3 i} \cdot 2^{3}\right)}\right)\right\} \\
& \geqslant_{L^{*}} \mathcal{T}_{i=0}^{m-1}\left\{\mathcal{P}^{\prime}{ }_{\alpha, \beta}(h(u, 0,0), \varepsilon)\right\} \geqslant_{L^{*}} \mathcal{P}^{\prime}{ }_{\alpha, \beta}(h(u, 0,0), \varepsilon)
\end{aligned}
$$

for all $u \in U$ and $\varepsilon>0$. Replacing $u$ by $2^{m^{\prime}} u$ in (3.10) and using (3.1), we get

$$
\mathcal{P}_{\alpha, \beta}\left(\frac{g\left(2^{m+m^{\prime}} u\right)}{2^{3\left(m+m^{\prime}\right)}}-\frac{g\left(2^{m^{\prime}} u\right)}{2^{3 m^{\prime}}}, \Sigma_{i=0}^{m-1} \frac{\tau^{i} \varepsilon}{24.2^{3\left(i+m^{\prime}\right)}}\right) \geqslant L^{*} \mathcal{P}^{\prime}{ }_{\alpha, \beta}\left(h(u, 0,0), \frac{\varepsilon}{\tau^{m^{\prime}}}\right)
$$

for all $u \in U, \varepsilon>0$, and $m, m^{\prime} \geqslant 0$. Replacing $\varepsilon$ by $\tau^{m^{\prime}} \varepsilon$ in (3.11), we get

$$
\mathcal{P}_{\alpha, \beta}\left(\frac{g\left(2^{m+m^{\prime}} u\right)}{2^{3\left(m+m^{\prime}\right)}}-\frac{g\left(2^{m^{\prime}} u\right)}{2^{3 m^{\prime}}}, \Sigma_{i=m^{\prime}}^{m+m^{\prime}-1} \frac{\tau^{i} \varepsilon}{24.2^{3 i}}\right) \geqslant_{L^{*}} \mathcal{P}^{\prime}{ }_{\alpha, \beta}(h(u, 0,0), \varepsilon)
$$

for all $u \in U, \varepsilon>0$, and $m, m^{\prime} \geqslant 0$. Using (IFN3) in (3.12), we get

$$
\mathcal{P}_{\alpha, \beta}\left(\frac{g\left(2^{m+m^{\prime}} u\right)}{2^{3\left(m+m^{\prime}\right)}}-\frac{g\left(2^{m^{\prime}} u\right)}{2^{3 m^{\prime}}}, \varepsilon\right) \geqslant L_{L^{*}} \mathcal{P}^{\prime}{ }_{\alpha, \beta}\left(h(u, 0,0), \frac{\varepsilon}{\sum_{i=m^{\prime}}^{m+m^{\prime}-1} \frac{\tau^{i} \varepsilon}{24.2^{3 i}}}\right)
$$

for all $u \in U, \varepsilon>0$ and $m, m^{\prime} \geqslant 0$. Since $0<\tau<2^{3}$ and $\sum_{i=0}^{m}\left(\frac{\tau}{2^{3}}\right)^{i}<\infty$. Thus $\left\{\frac{g\left(2^{m} u\right)}{2^{3 m}}\right\}$ is a Cauchy sequence in $V$. Since $\left(V, \mathcal{P}_{\alpha, \beta}, \mathcal{T}\right)$ is a complete IFNS, the sequence converges to some point $Q(u) \in V$. So, define the mapping $\mathrm{Q}: \mathrm{U} \rightarrow \mathrm{V}$ by

$$
\mathcal{P}_{\alpha, \beta}\left(\mathrm{Q}(\mathrm{u})-\frac{\mathrm{g}\left(2^{\gamma \mathrm{m}} \mathrm{u}\right)}{2^{\gamma 3 \mathrm{~m}}}\right) \longrightarrow 1_{\mathrm{L}^{*}} \text { as } \mathrm{m} \longrightarrow \infty, \quad \varepsilon>0
$$

for all $u \in U$. Letting $\mathrm{m}^{\prime} \rightarrow 0$ in (3.13), we obtain

$$
\mathcal{P}_{\alpha, \beta}\left(\frac{g\left(2^{m} u\right)}{2^{3 m}}-g(u), \varepsilon\right) \geqslant L^{*} \mathcal{P}^{\prime}{ }_{\alpha, \beta}\left(h(u, 0,0), \frac{\varepsilon}{\sum_{i=0}^{m-1} \frac{\tau^{i} \varepsilon}{24.2^{3 i}}}\right)
$$

for all $u \in U$ and $\varepsilon>0$. Letting $\mathrm{m} \rightarrow \infty$ in (3.14), we find

$$
\mathcal{P}_{\alpha, \beta}(g(u)-Q(u), \varepsilon) \geqslant_{L^{*}} \mathcal{P}_{\alpha, \beta}^{\prime}\left(h(u, 0,0), 3 \varepsilon\left(2^{3}-\tau\right)\right) .
$$

To show $Q$ satisfies (1.2), replacing $\left(\mathfrak{u}_{1}, \mathfrak{u}_{2}, u_{3}\right)$ by $\left(2^{m} u_{1}, 2^{m} u_{2}, 2^{m} u_{3}\right)$ in (3.3), respectively, we get

$$
\mathcal{P}_{\alpha, \beta}\left(\frac{1}{2^{3 m}} \operatorname{Dg}\left(\left(2^{m} u_{1}, 2^{m} u_{2}, 2^{m} u_{3}\right), \varepsilon\right) \geqslant L_{L^{*}} \mathcal{P}^{\prime}{ }_{\alpha, \beta}\left(h\left(2^{m} u_{1}, 2^{m} u_{2}, 2^{m} u_{3}\right), 2^{3 m} \varepsilon\right)\right.
$$

for all $u_{1}, u_{2}, u_{3} \in U$ and $\varepsilon>0$. Now,

$$
\begin{aligned}
\mathcal{P}_{\alpha, \beta}(Q & \left(2 u_{1}+u_{2}+u_{3}\right)-3 Q\left(u_{1}+u_{2}+u_{3}\right)-Q\left(-u_{1}+u_{2}+u_{3}\right) \\
& -2 Q\left(u_{1}+u_{2}\right)-2 Q\left(u_{1}+u_{3}\right)+6 Q\left(u_{1}-u_{2}\right)+6 Q\left(u_{1}-u_{3}\right) \\
& \left.+3 Q\left(u_{2}+u_{3}\right)-2 Q\left(2 u_{1}-u_{2}\right)-2 Q\left(2 u_{1}-u_{3}\right)+18 Q\left(u_{1}\right)+6 Q\left(u_{2}\right)+6 Q\left(u_{3}\right), \varepsilon\right) \\
\geqslant & L^{*} \mathcal{T}\left\{\mathcal{P}_{\alpha, \beta}\left(Q\left(2 u_{1}+u_{2}+u_{3}\right)-\frac{1}{2^{3 m}} g\left(2^{m}\left(2 u_{1}+u_{2}+u_{3}\right)\right), \frac{\varepsilon}{2^{3}}\right),\right.
\end{aligned}
$$




$$
\begin{aligned}
& \mathcal{P}_{\alpha, \beta}\left(-3 Q\left(u_{1}+u_{2}+u_{3}\right)+\frac{1}{2^{3 m}} 3 g\left(2^{m}\left(u_{1}+u_{2}+u_{3}\right)\right), \frac{\varepsilon}{2^{3}}\right), \\
& \mathcal{P}_{\alpha, \beta}\left(-\mathrm{Q}\left(-\mathfrak{u}_{1}+\mathfrak{u}_{2}+\mathfrak{u}_{3}\right)+\frac{1}{2^{3 m}} g\left(2^{m}\left(-u_{1}+u_{2}+u_{3}\right)\right), \frac{\varepsilon}{2^{3}}\right), \\
& \mathcal{P}_{\alpha, \beta}\left(-2 \mathrm{Q}\left(\mathrm{u}_{1}+\mathrm{u}_{2}\right)+\frac{1}{2^{3 m}} 2 \mathrm{~g}\left(2^{\mathrm{m}}\left(2 \mathrm{Q}\left(\mathrm{u}_{1}+\mathrm{u}_{2}\right)\right), \frac{\varepsilon}{2^{3}}\right),\right. \\
& \mathcal{P}_{\alpha, \beta}\left(-2 \mathrm{Q}\left(\mathrm{u}_{1}+\mathrm{u}_{3}\right)+\frac{1}{2^{3 \mathrm{~m}}} 2 \mathrm{~g}\left(2^{\mathrm{m}}\left(2 \mathrm{Q}\left(\mathrm{u}_{1}+\mathrm{u}_{3}\right)\right), \frac{\varepsilon}{2^{3}}\right),\right. \\
& \mathcal{P}_{\alpha, \beta}\left(6 \mathrm{Q}\left(\mathrm{u}_{1}-\mathrm{u}_{2}\right)-\frac{1}{2^{3 m}} 6 g\left(2^{\mathrm{m}}\left(\mathrm{u}_{1}-\mathrm{u}_{2}\right)\right), \frac{\varepsilon}{2^{3}}\right), \\
& \mathcal{P}_{\alpha, \beta}\left(6 \mathrm{Q}\left(\mathrm{u}_{1}-\mathrm{u}_{3}\right)-\frac{1}{2^{3 m}} 6 g\left(2^{\mathrm{m}}\left(\mathrm{u}_{1}-\mathrm{u}_{3}\right)\right), \frac{\varepsilon}{2^{3}}\right), \\
& \mathcal{P}_{\alpha, \beta}\left(3 Q\left(u_{2}+u_{3}\right)-\frac{1}{2^{3 m}} 3 g\left(2^{m}\left(u_{2}+u_{3}\right)\right), \frac{\varepsilon}{2^{3}}\right), \\
& \mathcal{P}_{\alpha, \beta}\left(-2 \mathrm{Q}\left(2 u_{1}-u_{2}\right)+\frac{1}{2^{3 m}} 2 g\left(2^{m}\left(2 u_{1}-u_{2}\right)\right), \frac{\varepsilon}{2^{3}}\right), \\
& \mathcal{P}_{\alpha, \beta}\left(-2 \mathrm{Q}\left(2 u_{1}-u_{3}\right)+\frac{1}{2^{3 m}} 2 g\left(2^{m}\left(2 u_{1}-u_{3}\right)\right), \frac{\varepsilon}{2^{3}}\right), \quad \mathcal{P}_{\alpha, \beta}\left(18 Q\left(u_{1}\right)-\frac{1}{2^{3 m}} 18 g\left(2^{m}\left(u_{1}\right)\right), \frac{\varepsilon}{2^{3}}\right), \\
& \mathcal{P}_{\alpha, \beta}\left(6 \mathrm{Q}\left(\mathrm{u}_{2}\right)-\frac{1}{2^{3 m}} 6 \mathrm{~g}\left(2^{\mathrm{m}}\left(\mathrm{u}_{2}\right)\right), \frac{\varepsilon}{2^{3}}\right), \mathcal{P}_{\alpha, \beta}\left(6 \mathrm{Q}\left(\mathrm{u}_{3}\right)-\frac{1}{2^{3 m}} 6 \mathrm{~g}\left(2^{\mathrm{m}}\left(\mathrm{u}_{3}\right)\right), \frac{\varepsilon}{2^{3}}\right), \\
& \mathcal{P}_{\alpha, \beta}\left(\frac{1}{2^{3 m}} g\left(2^{m}\left(2 u_{1}+u_{2}+u_{3}\right)\right)-\frac{1}{2^{3 m}} 3 g\left(2^{m}\left(u_{1}+u_{2}+u_{3}\right)\right)\right. \\
& -\frac{1}{2^{3 m}} g\left(2^{m}\left(-u_{1}+u_{2}+u_{3}\right)\right)-\frac{1}{2^{3 m}} 2 g\left(2^{m}\left(2 Q\left(u_{1}+u_{2}\right)\right)-\frac{1}{2^{3 m}} 2 g\left(2^{m}\left(2 Q\left(u_{1}+u_{3}\right)\right)\right.\right. \\
& +\frac{1}{2^{3 m}} 6 g\left(2^{m}\left(u_{1}-u_{2}\right)\right)+\frac{1}{2^{3 m}} 6 g\left(2^{m}\left(u_{1}-u_{3}\right)\right)+\frac{1}{2^{3 m}} 3 g\left(2^{m}\left(u_{2}+u_{3}\right)\right) \\
& -\frac{1}{2^{3 m}} 2 g\left(2^{m}\left(2 u_{1}-u_{2}\right)\right)-\frac{1}{2^{3 m}} 2 g\left(2^{m}\left(2 u_{1}-u_{3}\right)\right) \\
& \left.\left.+\frac{1}{2^{3 m}} 18 g\left(2^{m}\left(u_{1}\right)\right)+\frac{1}{2^{3 m}} 6 g\left(2^{m}\left(u_{2}\right)\right)+\frac{1}{2^{3 m}} 6 g\left(2^{m}\left(u_{3}\right)\right)\right)\right\}
\end{aligned}
$$

for all $\mathfrak{u}_{1}, \mathfrak{u}_{2}, u_{3} \in U$ and $\varepsilon>0$. Using (3.15) in (3.16), we obtain

$$
\begin{aligned}
\mathcal{P}_{\alpha, \beta}( & Q\left(2 u_{1}+u_{2}+u_{3}\right)-3 Q\left(u_{1}+u_{2}+u_{3}\right) \\
& -Q\left(-u_{1}+u_{2}+u_{3}\right)-2 Q\left(u_{1}+u_{2}\right)-2 Q\left(u_{1}+u_{3}\right)+6 Q\left(u_{1}-u_{2}\right) \\
& +6 Q\left(u_{1}-u_{3}\right)+3 Q\left(u_{2}+u_{3}\right)-2 Q\left(2 u_{1}-u_{2}\right)-2 Q\left(2 u_{1}-u_{3}\right) \\
& \left.+18 Q\left(u_{1}\right)+6 Q\left(u_{2}\right)+6 Q\left(u_{3}\right), \varepsilon\right) \geqslant_{L^{*}} \mathcal{T}\left\{1_{L^{*}}, 1_{L^{*}}, 1_{L^{*},} 1_{L^{*}}, 1_{L^{*}}, 1_{L^{*}}, 1_{L^{*}},\right. \\
& \left.1_{L^{*},} 1_{L^{*},} 1_{L^{*}}, 1_{L^{*}}, 1_{L^{*}}, 1_{L^{*}}, \mathcal{P}^{\prime}{ }_{\alpha, \beta}\left(h\left(2^{m} u_{1}, 2^{m} u_{2}, 2^{m} u_{3}\right), 2^{3 m} \varepsilon\right)\right\} \\
\geqslant L^{*} & \mathcal{P}^{\prime}{ }_{\alpha, \beta}\left(h\left(2^{m} u_{1}, 2^{m} u_{2}, 2^{m} u_{3}\right), 2^{3 m} \varepsilon\right)
\end{aligned}
$$

for all $u_{1}, u_{2}, u_{3} \in U$ and $\varepsilon>0$. Letting $m \rightarrow \infty$ in (3.17) and using (3.2), we observe that

$$
\begin{aligned}
& \mathcal{P}_{\alpha, \beta}\left(Q\left(2 u_{1}+u_{2}+u_{3}\right)-3 Q\left(u_{1}+u_{2}+u_{3}\right)-Q\left(-u_{1}+u_{2}+u_{3}\right)-2 Q\left(u_{1}+u_{2}\right)\right. \\
& -2 Q\left(u_{1}+u_{3}\right)+6 Q\left(u_{1}-u_{2}\right)+6 Q\left(u_{1}-u_{3}\right)+3 Q\left(u_{2}+u_{3}\right)-2 Q\left(2 u_{1}-u_{2}\right) \\
& \left.-2 Q\left(2 u_{1}-u_{3}\right)+18 Q\left(u_{1}\right)+6 Q\left(u_{2}\right)+6 Q\left(u_{3}\right), \varepsilon\right)=1_{L^{*}}
\end{aligned}
$$

for all $u_{1}, u_{2}, u_{3} \in U$ and $\varepsilon>0$. Using (IFN2) in the above inequality, which gives

$$
\begin{aligned}
\mathrm{Q}\left(2 \mathrm{u}_{1}+\mathrm{u}_{2}+\mathrm{u}_{3}\right)= & 3 \mathrm{Q}\left(\mathrm{u}_{1}+\mathrm{u}_{2}+\mathrm{u}_{3}\right)+\mathrm{Q}\left(-\mathrm{u}_{1}+\mathrm{u}_{2}+\mathrm{u}_{3}\right)+2 \mathrm{Q}\left(\mathrm{u}_{1}+\mathrm{u}_{2}\right) \\
& +2 \mathrm{Q}\left(\mathrm{u}_{1}+\mathrm{u}_{3}\right)-6 \mathrm{Q}\left(\mathrm{u}_{1}-\mathrm{u}_{2}\right)-6 \mathrm{Q}\left(\mathrm{u}_{1}-\mathrm{u}_{3}\right)-3 \mathrm{Q}\left(\mathrm{u}_{2}+\mathrm{u}_{3}\right) \\
& +2 \mathrm{Q}\left(2 \mathrm{u}_{1}-\mathrm{u}_{2}\right)+2 \mathrm{Q}\left(2 \mathrm{u}_{1}-\mathrm{u}_{3}\right)-18 \mathrm{Q}\left(\mathrm{u}_{1}\right)-6 \mathrm{Q}\left(\mathrm{u}_{2}\right)-6 \mathrm{Q}\left(\mathrm{u}_{3}\right)
\end{aligned}
$$


for all $u_{1}, u_{2}, u_{3} \in U$. Hence $Q$ satisfies the cubic functional equation (1.2). In order to show $Q(u)$ is unique, let $\mathrm{Q}^{\prime}(\mathrm{u})$ be another cubic mapping satisfying (1.2) and (3.4). Hence

$$
\begin{aligned}
\mathcal{P}_{\alpha, \beta}\left(\mathrm{Q}(\mathrm{u})-\mathrm{Q}^{\prime}(\mathrm{u}), \varepsilon\right) & =\mathcal{P}_{\alpha, \beta}\left(\frac{\mathrm{Q}\left(2^{\mathrm{m}} \mathrm{u}\right)}{2^{3 \mathrm{~m}}}-\frac{\mathrm{Q}^{\prime}\left(2^{\mathrm{m}} \mathrm{u}\right)}{\left.2^{3 \mathrm{~m}}, \varepsilon\right)}\right. \\
& \geqslant_{\mathrm{L}^{*}} \mathcal{T}\left\{\mathrm{P}_{\alpha, \beta}\left(\frac{\mathrm{Q}\left(2^{\mathrm{m}} \mathrm{u}\right)}{2^{3 \mathrm{~m}}}-\frac{\mathrm{g}\left(2^{\mathrm{m}} \mathrm{u}\right)}{2^{3 \mathrm{~m}}}, \frac{\varepsilon}{2}\right), \mathrm{P}_{\alpha, \beta}\left(\frac{\mathrm{g}\left(2^{\mathrm{m}} \mathrm{u}\right)}{2^{3 \mathrm{~m}}}-\frac{\mathrm{Q}^{\prime}\left(2^{\mathrm{m}} \mathrm{u}\right)}{2^{3 \mathrm{~m}}}, \frac{\varepsilon}{2}\right)\right\} \\
& \geqslant_{\mathrm{L}^{*}} \mathrm{P}_{\alpha, \beta}^{\prime}\left(\mathrm{h}\left(2^{\mathrm{m}} \mathrm{u}, 0,0\right), \frac{3\left(2^{\mathrm{m}}\right) \varepsilon\left(2^{3}-\tau\right)}{2}\right) \\
& \geqslant_{\mathrm{L}^{*}} \mathrm{P}_{\alpha, \beta}^{\prime}\left(\mathrm{h}(\mathrm{u}, 0,0), \frac{3\left(2^{\mathrm{m}}\right) \mathcal{\varepsilon}\left(2^{3}-\tau\right)}{2 \cdot \tau^{\mathrm{m}}}\right)
\end{aligned}
$$

for all $u \in U$ and $\varepsilon>0$. Since

$$
\lim _{m \rightarrow \infty} \frac{3\left(2^{m}\right) \varepsilon\left(2^{3}-\tau\right)}{2 \cdot \tau^{m}}=\infty
$$

we get

$$
\lim _{m \rightarrow \infty} P_{\alpha, \beta}^{\prime}\left(h(u, 0,0), \frac{3\left(2^{m}\right) \varepsilon\left(2^{3}-\tau\right)}{2 \cdot \tau^{m}}\right)=1_{L^{*}}
$$

Thus,

$$
\mathrm{P}_{\alpha, \beta}\left(\mathrm{Q}(\mathrm{u})-\mathrm{Q}^{\prime}(\mathrm{u}), \varepsilon\right)=1_{\mathrm{L}^{*}}
$$

for all $u \in U$ and $\varepsilon>0$, hence $Q(u)=Q^{\prime}(u)$. Therefore, $Q(u)$ is unique. For $\gamma=-1$, we can show the result by a similar method. This completes the proof of the theorem.

From above theorem, we have the following corollary concerning the Hyers-Ulam-Rassias and JMRassias stabilities for the functional equation (1.2).

Corollary 3.2. Assume that a function $\mathrm{g}: \mathrm{U} \rightarrow \mathrm{V}$ satisfies the inequality

$$
P_{\alpha, \beta}\left(\operatorname{Dg}\left(u_{1}, u_{2}, u_{3}\right), \varepsilon\right) \geqslant L^{*}\left\{\begin{array}{l}
P_{\alpha, \beta}^{\prime}\left(\delta \Sigma_{i=1}^{3}\left\|u_{i}\right\|^{s}, \varepsilon\right), \\
P_{\alpha, \beta}^{\prime}\left(\delta\left(\Pi_{i=1}^{3}\left\|u_{i}\right\|^{s}+\Sigma_{i=1}^{3}\left\|u_{i}\right\|^{3 s}\right), \varepsilon\right)
\end{array}\right.
$$

for all $\mathrm{u}_{1}, \mathfrak{u}_{2}, \mathfrak{u}_{3} \in \mathrm{U}$ and $\varepsilon>0$, where $\delta$, s are constants with $\delta>0$. Then there exists a unique cubic mapping $\mathrm{Q}: \mathrm{U} \rightarrow \mathrm{V}$ such that

$$
P_{\alpha, \beta}\left(D g\left(u_{1}, u_{2}, u_{3}\right), \varepsilon\right) \geqslant L^{*} \begin{cases}P_{\alpha, \beta}^{\prime}\left(\delta\|u\|^{s}, 3 \varepsilon \mid 2^{3}-2^{s}\right), & s \neq 3 \\ P_{\alpha, \beta}^{\prime}\left(\delta\left\|u_{i}\right\|^{3 s}, 3 \varepsilon\left|2^{3}-2^{m s}\right|\right), & s \neq \frac{3}{m}\end{cases}
$$

for all $\mathrm{u} \in \mathrm{U}$ and $\varepsilon>0$. If we define

$$
\psi\left(u_{1}, u_{2}, u_{3}\right)=\left\{\begin{array}{l}
\delta \Sigma_{i=1}^{3}\left\|u_{i}\right\|^{s} \\
\delta\left(\Pi_{i=1}^{3}\left\|u_{i}\right\|^{s}+\delta \Sigma_{i=1}^{3}\left\|u_{i}\right\|^{3 s}\right),
\end{array}\right.
$$

then the corollary is followed from above theorem, by taking $\tau=\left\{\begin{array}{l}2^{s}, \\ 2^{3 s}\end{array}\right.$.

\section{Stability result for the functional equation: fixed point method}

In this segment, we examine the generalized Ulam-Hyers stability of the functional equation (1.2) in IFNS using fixed point method. Now, we recall the fundamental results in fixed point theory. 
Theorem 4.1 (The alternative of fixed point, [12])). Suppose that for a complete generalized metric space (U, $\tau)$ and a strictly contractive mapping $\mathcal{T}: \mathrm{U} \rightarrow \mathrm{U}$ with Lipschitz constant $\mathrm{L}$. Then, for each given element $\mathrm{u} \in \mathrm{U}$, either

$$
\tau\left(\mathcal{T}^{\mathrm{m}} \mathfrak{u}, \mathcal{T}^{\mathrm{m}+1} \mathfrak{u}\right)=\infty, \forall \mathrm{m} \geqslant 0,
$$

or there exists a natural number $\mathrm{m}_{0}$ such that

(1) $\tau\left(\mathcal{T}^{\mathrm{m}} \mathfrak{u}, \mathcal{T}^{\mathrm{m}+1} \mathrm{u}\right)<\infty$ for all $\mathrm{m} \geqslant \mathrm{m}_{0}$;

(2) The sequence $\left(\mathcal{T}^{\mathrm{m}} \mathrm{u}\right)$ is convergent to a fixed point $v *$ of $\mathcal{T}$;

(3) $v *$ is the unique fixed point of $\mathcal{T}$ in the set $\mathrm{V}=\left\{v \in \mathrm{U}: \tau\left(\mathcal{T}^{\mathrm{m}_{0}} \mathrm{u}, v\right)<\infty\right\}$;

(4) $\tau(\nu *, v) \leqslant \frac{1}{1-\mathrm{L}} \tau(\nu, \mathcal{T} v)$ for all $v \in \mathrm{V}$.

Proof. For proving the stability result, we define: $\xi_{i}$ is a constant such that

$$
\xi_{i}= \begin{cases}2, & \text { if } i=0, \\ \frac{1}{2}, & \text { if } i=1,\end{cases}
$$

and $\Lambda$ is the set such that $\Lambda=\{\mu \mid \mu: U \rightarrow V, \mu(0)=0\}$.

Theorem 4.2. Let $\mathrm{g}: \mathrm{U} \longrightarrow \mathrm{V}$ be a mapping for which there exist a function $\mathrm{h}: \mathrm{U}^{3} \rightarrow z$ with the condition

$$
\lim _{l \rightarrow \infty} P_{\alpha, \beta}^{\prime}\left(h\left(\xi_{i}^{l} u_{1}, \xi_{i}^{l} u_{2}, \xi_{i}^{l} u_{3}\right), \xi_{i}^{3 l} \varepsilon\right)=1_{L^{*}}
$$

for all $\mathrm{u}_{1}, \mathrm{u}_{2}, \mathrm{u}_{3} \in \mathrm{U}, \varepsilon>0$ and satisfying the inequality

$$
P_{\alpha, \beta}\left(D g\left(u_{1}, u_{2}, u_{3}\right), \varepsilon\right) \geqslant_{L^{*}} P_{\alpha, \beta}\left(h\left(u_{1}, u_{2}, u_{3}\right), \varepsilon\right)
$$

for all $\mathrm{u}_{1}, \mathrm{u}_{2}, \mathrm{u}_{3} \in \mathrm{U}, \varepsilon>0$. If there exists $\mathrm{L}=\mathrm{L}(\mathfrak{i})$ such that the function

$$
u \rightarrow \gamma(u)=\frac{1}{3} h\left(\frac{u}{2}, 0,0\right)
$$

has the property

$$
\mathrm{P}_{\alpha, \beta}^{\prime}\left(\mathrm{L} \frac{1}{\xi_{i}^{3}} \gamma\left(\xi_{i} u\right), \varepsilon\right)=\mathrm{P}_{\alpha, \beta}^{\prime}(\gamma(u), \varepsilon)
$$

for all $\mathrm{u} \in \mathrm{U}, \varepsilon>0$, then there exists unique cubic function $\mathrm{Q}: \mathrm{U} \rightarrow \mathrm{V}$ satisfying the functional equation (1.2) and

$$
P_{\alpha, \beta}(g(u)-Q(u), \varepsilon) \geqslant L^{*} P_{\alpha, \beta}^{\prime}\left(\gamma(u), \frac{L^{1-i}}{1-L} \varepsilon\right)
$$

for all $\mathrm{u} \in \mathrm{U}, \varepsilon>0$.

Proof. Let $\tau$ be a general metric on $\Lambda$, such that

$$
\tau(\mu, v)=\inf \left\{M \in(0, \infty) \mid P_{\alpha, \beta}(\mu(u)-v(u), \varepsilon) \geqslant L^{*} P_{\alpha, \beta}^{\prime}(\gamma(u), M \varepsilon), u \in U, \varepsilon>0\right\} .
$$

It is simple to verify that $(\Lambda, \tau)$ is complete. Define $T: \Lambda \rightarrow \Lambda$ by

$$
\mathrm{T}(\mu(\mathrm{u}))=\frac{1}{\xi_{i}^{3}} \mu\left(\xi_{i} u\right)
$$

for all $u \in U$. For $\mu, v \in \Lambda$, we have $\tau(\mu, v) \leqslant M$,

$$
P_{\alpha, \beta}(\mu(u)-v(u), \varepsilon) \geqslant_{L^{*}} P_{\alpha, \beta}^{\prime}(\gamma(u), M \varepsilon),
$$




$$
\begin{aligned}
P_{\alpha, \beta}\left(\frac{\mu\left(\xi_{i} u\right)}{\xi_{i}^{3}}-\frac{v\left(\xi_{i} u\right)}{\xi_{i}^{3}}, \varepsilon\right) & \geqslant L^{*} P_{\alpha, \beta}^{\prime}\left(\gamma\left(\xi_{i} u\right), M \xi_{i}^{3} \varepsilon\right), \\
P_{\alpha, \beta}(\mathcal{T} \mu(u)-\mathcal{T} v(u), \varepsilon) & \geqslant L^{*} P_{\alpha, \beta}^{\prime}(\gamma(u), M L \varepsilon), \\
\tau(\mathcal{T} \mu(u), \mathcal{T} v(u), \varepsilon) & \leqslant M L \\
\tau(\mathcal{T} \mu, \mathcal{T} v) & \leqslant L \tau(\mu, v)
\end{aligned}
$$

for all $\mu, \nu \in \Lambda$. Therefore $\mathcal{T}$ is strictly contractive mapping on $\Lambda$ with Lipschitz constant L. Replacing $\left(u_{1}, u_{2}, u_{3}\right)$ by $(u, 0,0)$ in $(4.1)$, we obtain

$$
P_{\alpha, \beta}(3 g(2 u)-24 g(u), \varepsilon) \geqslant L^{*} P_{\alpha, \beta}^{\prime}(h(u, 0,0), \varepsilon) .
$$

Using (IFN2) in (4.3), we obtain

$$
P_{\alpha, \beta}\left(\frac{g(2 u)}{2^{3}}-g(u), \varepsilon\right) \geqslant L^{*} P_{\alpha, \beta}^{\prime}(h(u, 0,0), 24 \varepsilon)
$$

with the help of (4.2), when $i=0$, it follows from (4.4), we get

$$
\begin{aligned}
P_{\alpha, \beta}\left(\frac{g(2 u)}{2^{3}}-g(u), \varepsilon\right) & \geqslant L^{*} P_{\alpha, \beta}^{\prime}(\gamma(u), L \varepsilon), \\
\tau(\mathcal{T} g, g) & \leqslant L=L^{\prime}=L^{1-i}
\end{aligned}
$$

Replacing $u$ by $\frac{u}{2}$ in (4.3), we get

$$
P_{\alpha, \beta}\left(g(u)-2^{3} g\left(\frac{u}{2}\right), \varepsilon\right) \geqslant_{L^{*}} P_{\alpha, \beta}^{\prime}\left(h\left(\frac{u}{2}, 0,0\right), 3 \varepsilon\right)
$$

with the help of (4.2) when $i=1$, it follows from (4.6), we get

$$
\begin{aligned}
P_{\alpha, \beta}\left(g(u)-2^{3} g\left(\frac{u}{2}\right), \varepsilon\right) & \geqslant L^{*} P_{\alpha, \beta}^{\prime}(\gamma(u), \varepsilon), \\
\tau(g, \mathcal{T g}) & \leqslant 1=L^{0}=L^{1-i} .
\end{aligned}
$$

Then from (4.5) and (4.7), we can conclude

$$
\tau(g, \mathcal{T} g) \leqslant \mathrm{L}^{1-i}<\infty .
$$

Now from the fixed point alternative in both cases, it follows that there exists a fixed point $\mathrm{Q}$ of $\mathcal{T}$ in $\Lambda$ such that

$$
\lim _{m \rightarrow \infty} P_{\alpha, \beta}\left(\frac{g\left(\xi_{i}^{m} u\right)}{\xi_{i}^{m}}-Q(u), \varepsilon\right) \rightarrow 1_{L^{*}}
$$

for all $u \in U, \varepsilon>0$. Replacing $\left(u_{1}, u_{2}, u_{3}\right)$ by $\left(\xi_{i} u_{1}, \xi_{i} u_{2}, \xi_{i} u_{3}\right)$ in (4.1), we get

$$
P_{\alpha, \beta}\left(\frac{1}{\xi_{i}^{3 m}} \operatorname{Dg}\left(\xi_{i} u_{1}, \xi_{i} u_{2}, \xi_{i} u_{3}\right), \varepsilon\right) \geqslant L^{*} P_{\alpha, \beta}^{\prime}\left(h\left(\xi_{i} u_{1}, \xi_{i} u_{2}, \xi_{i} u_{3}\right), \xi_{i}^{3 m} \varepsilon\right)
$$

for all $u_{1}, u_{2}, u_{3} \in U$ and $\varepsilon>0$. By proceeding the same procedure as in the above theorem (3.1), we can show the function $\mathrm{Q}: \mathrm{U} \rightarrow \mathrm{V}$ satisfies the functional equation (1.2). By fixed point alternative, since $\mathrm{Q}$ is unique fixed point of $\mathcal{T}$ in the set

$$
\Gamma=\{g \in \Lambda \mid \tau(g, Q)<\infty\}
$$

hence $Q$ is a unique function such that

$$
P_{\alpha, \beta}(g(u)-Q(u), \varepsilon) \geqslant_{L^{*}} P_{\alpha, \beta}^{\prime}(\gamma(u), M \varepsilon)
$$


for all $u \in U, \varepsilon>0$, and $M>0$. Again using the fixed point alternative, we obtain

$$
\tau(g, Q) \leqslant \frac{1}{1-L} \tau(g, \mathcal{T g}), \quad \tau(g, Q) \leqslant \frac{L^{1-i}}{1-L^{\prime}}, \quad P_{\alpha, \beta}(g(u)-Q(u), \varepsilon) \geqslant L^{*} P_{\alpha, \beta}^{\prime}\left(\gamma(u), \frac{L^{1-i}}{1-L} \varepsilon\right)
$$

for all $u \in U, \varepsilon>0$. This completes the proof of the theorem.

From above theorem, we have the following corollary concerning the stability for the functional equation (1.2).

Corollary 4.3. Assume that a function $\mathrm{g}: \mathrm{U} \rightarrow \mathrm{V}$ satisfies the inequality

$$
P_{\alpha, \beta}\left(\operatorname{Dg}\left(u_{1}, u_{2}, u_{3}\right)\right) \geqslant\left\{\begin{array}{l}
P_{\alpha, \beta}^{\prime}\left(\xi \Sigma_{i=1}^{3}\left\|u_{i}\right\|^{s}, \varepsilon\right) \\
P_{\alpha, \beta}^{\prime}\left(\xi \Pi_{i=1}^{3}\left\|u_{i}\right\|^{s}+\Sigma_{i=1}^{3}\left\|u_{i}\right\|^{3 s}, \varepsilon\right)
\end{array}\right.
$$

for all $\mathrm{u}_{1}, \mathrm{u}_{2}, \mathrm{u}_{3} \in \mathrm{U}$ and $\varepsilon>0$, where $\varepsilon$, s are constants with $\varepsilon>0$. Then there exists a unique cubic mapping $\mathrm{Q}: \mathrm{U} \rightarrow \mathrm{V}$ such that

$$
P_{\alpha, \beta}(g(u)-Q(u), \varepsilon) \geqslant L^{*} \begin{cases}P_{\alpha, \beta}^{\prime}\left(\xi\|u\|^{s}, \frac{2^{s+2}}{2^{3}-2^{s}} \varepsilon\right), & s<3 \text { or } s>3, \\ P_{\alpha, \beta}^{\prime}\left(\xi\|u\|^{3 s}, \frac{2^{3 s+2}}{2^{3}-2^{m s}} \varepsilon\right), & s<\frac{3}{m} \text { or } s>\frac{3}{m}\end{cases}
$$

for all $\mathrm{u} \in \mathrm{U}$ and $\varepsilon>0$.

\section{Acknowledgment}

The authors thank the referees for their valuable comments and suggestions.

\section{References}

[1] R. P. Agarwal, Y. J. Cho, R. Saadati, S. Wang, Nonlinear L-fuzzy stability of cubic functional equations, J. Inequal. Appl., 2012 (2012), 19 pages. 1

[2] M. Amini, R. Saadati, Topics in fuzzy metric space, J. Fuzzy Math., 11 (2003), 765-768.

[3] M. Arunkumar, S. Karthikeyan, Intuitionistic Random stability of a quadratic functional equation originating from the sum of the medians of a triangle, Int. J. Difference Equ., 12 (2017), 55-70.

[4] M. Arunkumar, S. Karthikeyan, Fuzzy Banach Algebra stability of reciprocal quadratic functional equation via fixed point approach, Int. J. Pure Appl. Math., 119 (2018), 31-39. 1

[5] R. C. Ashish, M. Kumar, Hyers-Ulam-Rassias stability of quadratic functional equations in multi Banach Spaces, Int. J. Pure. App. M., 86 (2013), 621-631. 1

[6] K. T. Atanassov, Intuitionistic fuzzy sets, Fuzzy Sets and Systems, 20 (1986), 87-96. 1.2, 1.3

[7] A. Bodaghi, Intuitionistic fuzzy stability of the generalized forms of cubic and quartic functional equations, J. Intell. Fuzzy Syst., 30 (2016), 2309-2317. 1

[8] A. Bodaghi, P. Narasimman, Stability of the general form of quadratic-quartic functional equations in non-Archimedean L-fuzzy normed spaces, Tbilisi Math. J., 11 (2018), 15-29. 1

[9] R. Chugh, A. Ashish, M. Kumar, On the stability of quartic functional equations via fixed point and direct method, Int. J. Comput. Appl., 40 (2012), 23-28. 1

[10] G. Deschrijver, E. E. Kerre, On the relationship between some extensions of fuzzy set theory, Fuzzy Sets and Systems, 133 (2003), 227-235. 1.1

[11] G. Deschrijver, D. O’Regan, R. Saadati, S. M. Vaezpour, L-Fuzzy Euclidean normed spaces and compactness, Chaos Solitons Fractals, 42 (2009), 40-45. 1

[12] J. B. Diaz, B. Margolis, A fixed point theorem of the alternative for contractions on a generalized complete metric space, Bull. Amer. Math. Soc., 126 (1968), 305-309. 1, 4.1

[13] P. Găvruţa, A generalization of the Hyers-Ulam-Rassias stability of approximately additive mappings, J. Math. Anal. Appl., 184 (1994), 431-436. 1

[14] V. Govindan, C. Park, S. Pinelas, S. Baskaran, Solution of a 3-D cubic functional equation and its stability, AIMS Math., 5 (2020), 1693-1705. 1

[15] D. H. Hyers, On the stability of the linear functional equation, Proc. Nat. Acad. Sci. U.S.A., 27 (1941), 222-224. 1

[16] K.-W. Jun, H.-M. Kim, The generalized Hyers-Ulam-Rassias stability of a cubic functional equation, J. Math. Anal. Appl., 274 (2002), 267-278. 1 
[17] A. K. Katsaras, Fuzzy topological vector spaces. II, Fuzzy Sets and Systems, 12 (1984), 143-154.

[18] G. H. Kim, Y.-H. Lee, Stability of an additive-quadratic-quartic functional equation, Demonstr. Math., 53 (2020), 1-7. 1

[19] S. O. Kim, J. M. Rassias, Stability of the Apollonius type additive functional equation in modular space and fuzzy Banach spaces, Mathematics, 2019 (2019), 17 pages.

[20] M. Kumar, R. Chugh, A. Ashish, Hyers-Ulam-Rassias stability of quadratic functional equations in 2-Banach Spaces, Int. J. Comput. Appl., 63 (2013), 1-4. 1

[21] Y.-H. Lee, S.-M. Jung, A fixed point approach to the stability of an additive-quadratic-cubic-quartic functional equation, J. Funct. Spaces, 2016 (2016), 7 pages. 1

[22] T. M. Rassias, On the stability of the linear mapping in Banach spaces, Proc. Amer. Math. Soc., 72 (1978), 297-300. 1

[23] J. M. Rassias, Solution of the Ulam stability problem for quartic mappings, Glas. Mat. Ser. III, 34 (1999), 243-252. 1

[24] J. M. Rassias, Solution of the Ulam stability problem for cubic mappings, Glas. Mat. Ser. III, 36 (2001), 63--72. 1

[25] K. Ravi, J. M. Rassias, B. V. S. Kumar, A. Bodaghi, Intuitionistic fuzzy stability of a reciprocal-quadratic functional equation, Int. J. Appl. Sci. Math., 1 (2014), 9-14. 1

[26] R. Saadati, J. H. Park, On the intuitionistic fuzzy topological spaces, Chaos Solitons Fractals, 27 (2006), 331-344.

[27] R. Saadati, S. M. Vaezpour, Some results on fuzzy Banach spaces, J. Appl. Math. Comput., 17 (2005), 475-484. 1

[28] S. Shakeri, Intuitionistic fuzzy stability of Jensen type mapping, J. Nonlinear Sci. Appl., 2 (2009), 105-112. 1.4, 1.5, 1.6

[29] S. M. Ulam, A Collection of Mathematical Problems, Interscience Publishers, New York-London, (1940). 1

[30] L. A. Zadeh, Fuzzy sets, Inf. Control, 8 (1965), 338-353. 1 